\title{
Ion Irradiation Shaping of Dense Two-dimensional Arrays of Au Nanoparticles Embedded in Silica Studied via TEM
}

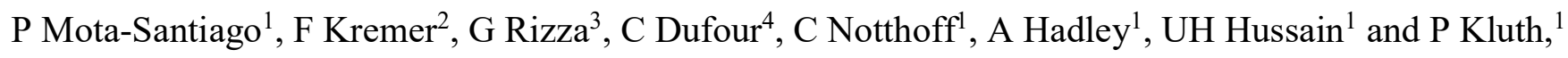 \\ 1. Department of Electronic Materials Engineering, Research School of Physics and Engineering, \\ Australian National University, Canberra, Australia. \\ 2. Centre for Advanced Microscopy, The Australian National University, Australia. \\ 3. Ecole Polytechnique, Laboratoire des Solides Irradies (LSI) CEA/DSM/IRAMIS, CNRS 91128 \\ Palaiseau Cedex, France. \\ 4. CIMAP/CEA/CNRS/ENSICAEN/ Universite de Caen, 6 Boulevard du Marechal Juin, 1405 Caen \\ Cedex 4, France.
}

The production of highly anisotropic metal nanoparticles (NPs) in transparent dielectrics is of special interest due to the possibility of exploiting their linear and non-linear optical response. Controlling size, shape, orientation and particle density represents a way to tailor their optical response. This is therefore a crucial step for incorporation into future plasmonic devices. In this contribution we report on a transmission electron microscopy study of gold elongated NPs embedded produced via deposition and swift heavy ion irradiation.

The sample fabrication process consists of the deposition of a $\sim 140 \mathrm{~nm}$ thick amorphous silicon nitride layer followed by a thin Au layer deposited via thermal evaporation. The Au film was then covered with $\mathrm{a} \sim 145 \mathrm{~nm}$ thick amorphous silicon dioxide layer and a final $\sim 140 \mathrm{~nm}$ thick silicon nitride capping layer. Rapid thermal annealing (RTA) at $950{ }^{\circ} \mathrm{C}$ in $\mathrm{N}_{2}$ atmosphere was then employed to induce the breakdown of the continuous $\mathrm{Au}$ film into a system of discrete metal NPs [Fig. 1(b)]. The ion beam shaping process was performed by swift heavy ion irradiation with $185 \mathrm{keV} \mathrm{Au}$ ions at fluences ranging from $1.0 \times 10^{13}$ $3.0 \times 10^{14}$ ions $/ \mathrm{cm}^{2}$ in normal incidence [Fig. 1(c)].

The transmission electron microscopy (TEM) results demonstrate that after the RTA an array of Au NPs has been formed at the interface between the bottom silicon nitride layer and the silicon dioxide layer [Fig. 2(a)]. The particle size distribution is characterized by a mean particle size of $\sim 35.0 \pm 9.0 \mathrm{~nm}$. Upon ion irradiation nanoparticle elongation is already noticeable for fluences as low as $3.0 \times 10^{13}$ ions $/ \mathrm{cm}^{2}$. The elongation process progress with increasing irradiation fluence. We observe, however, that even after irradiation with $3.0 \times 10^{14}$ ions $/ \mathrm{cm}^{2}$ the elongated NPs are still confined within the silicon dioxide layer [Fig. 2(b)].

The obtained results are discussed based on the Thermal Spike model. During ion irradiation, intense electronic excitations are produced in a narrow region surrounding the ion path. The interaction between the ions and the electrons in the material generate electron cascades moving radially outwards, interacting with atoms in the lattice via electron-phonon coupling. The energy deposited in the lattice results in a fast increase in the local temperature, hence Thermal Spike, that is capable to exceed the melting temperature on the material in a narrow region surrounding the ion pathway [1]. In this scenario, the short-lived thermal spike in silicon nitride limits the shaping of the $\mathrm{Au}$ nanoparticles in this region hence confining the elongation process to the silicon dioxide layer. 
In conclusion, the difference in thermophysical properties between silicon nitride and silicon dioxide, particularly thermal conductivity and electron phonon coupling, can be used to confine the ion shaping process to the silicon dioxide intermediate layer. Such confinement represents a step forward towards the design of very thin layers with controlled anisotropic metallic nanoparticles for plasmonic or photonic applications [2].

References:

[1] P Mota-Santiago et al., Nanotechnology 29 (2018), p. 144004.

[2] The authors would like to acknowledge the support from the ARC, AMMRF, ANFF and NCRIS. $185 \mathrm{keV}$ Au ions
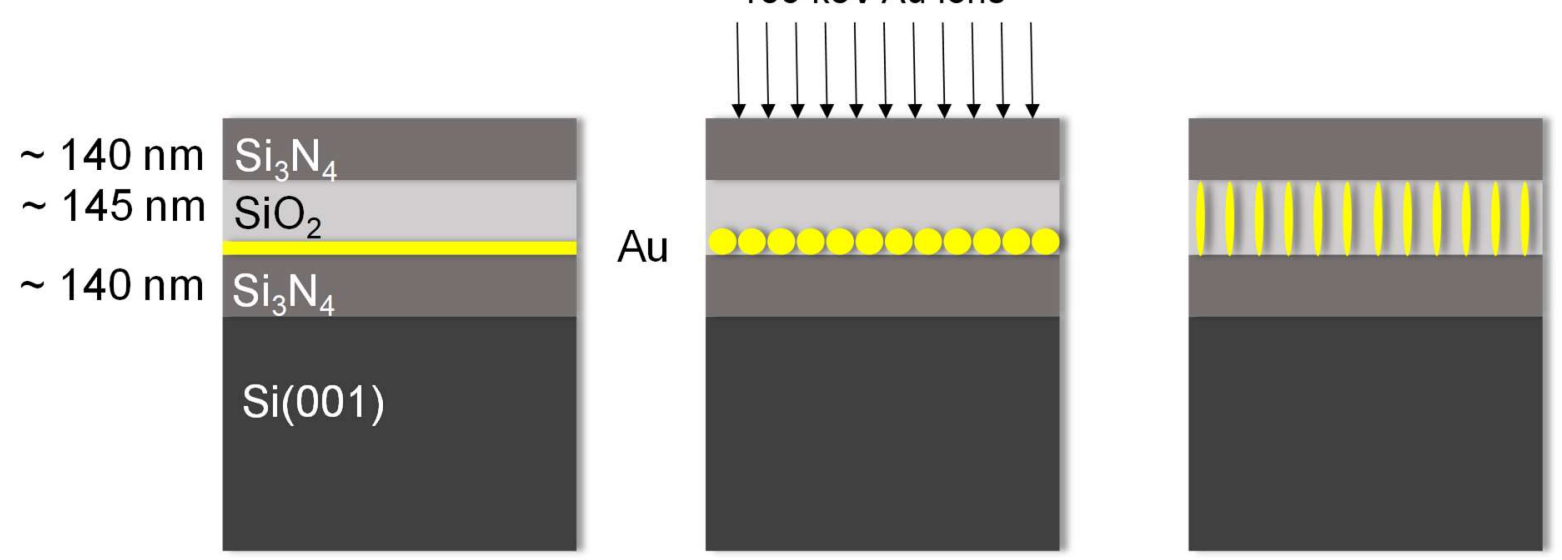

Figure 1. Scheme representing the sample fabrication process. First the films are deposited onto a $\mathrm{Si}(001)$ substrate. A post deposition RTA treatment is performed to produce the Au NPs (middle panel). Finally, high energy ion irradiation is performed to induce NP shaping.
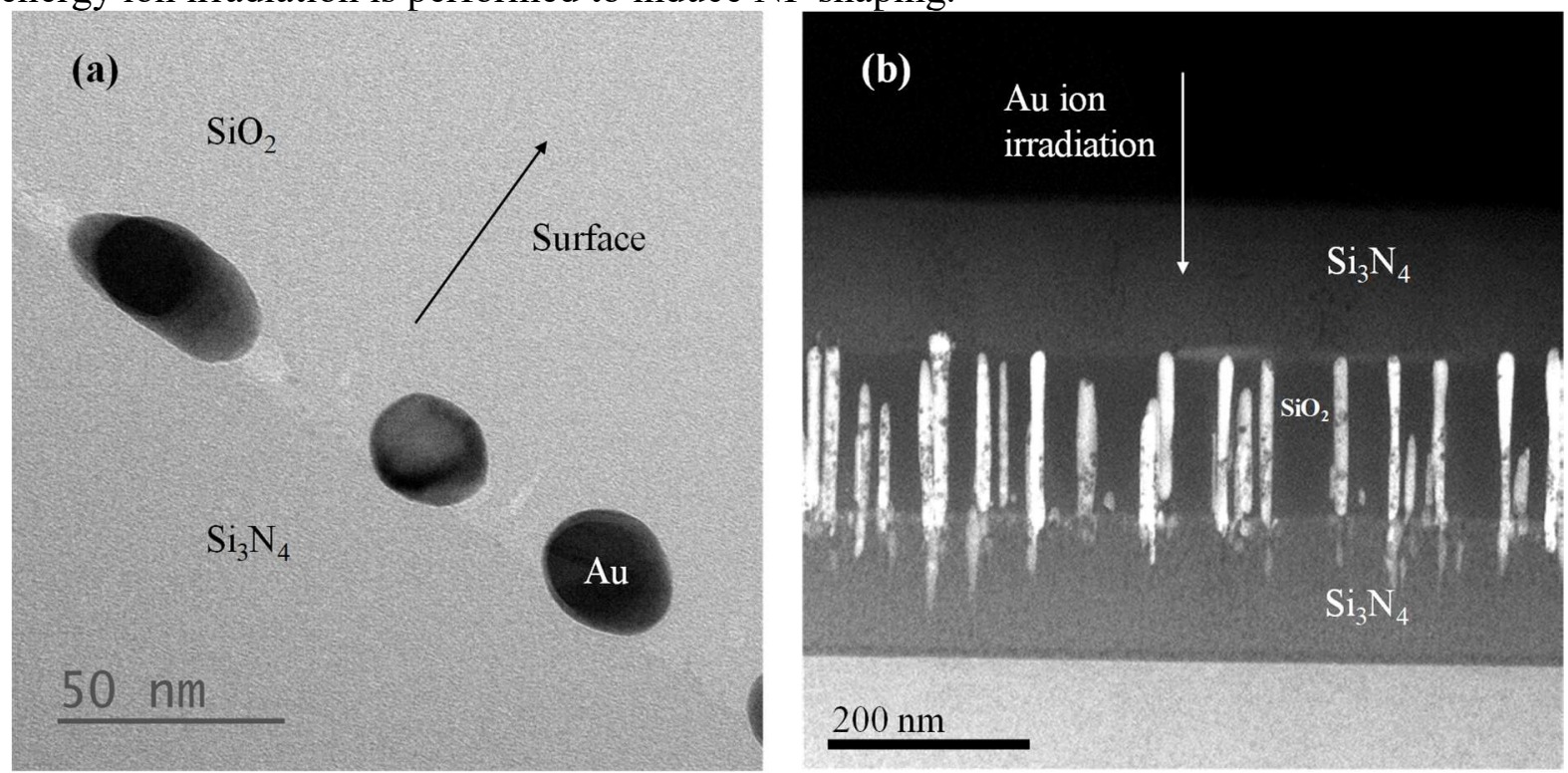

Figure 2. (a) Cross session TEM displaying the Au nanoparticles after deposition and RTA treatment. (b) High angular annular dark field image of the Au elongated nanoparticles after irradiation with $3.0 \times 10^{14}$ $\mathrm{Au}$ ions $/ \mathrm{cm}^{2}$ at $185 \mathrm{keV}$. The images were obtained the sample oriented towards the [110] zone axis from Si parallel to the (001) plane from the Si surface. 